Page 1 of 2 Proj.

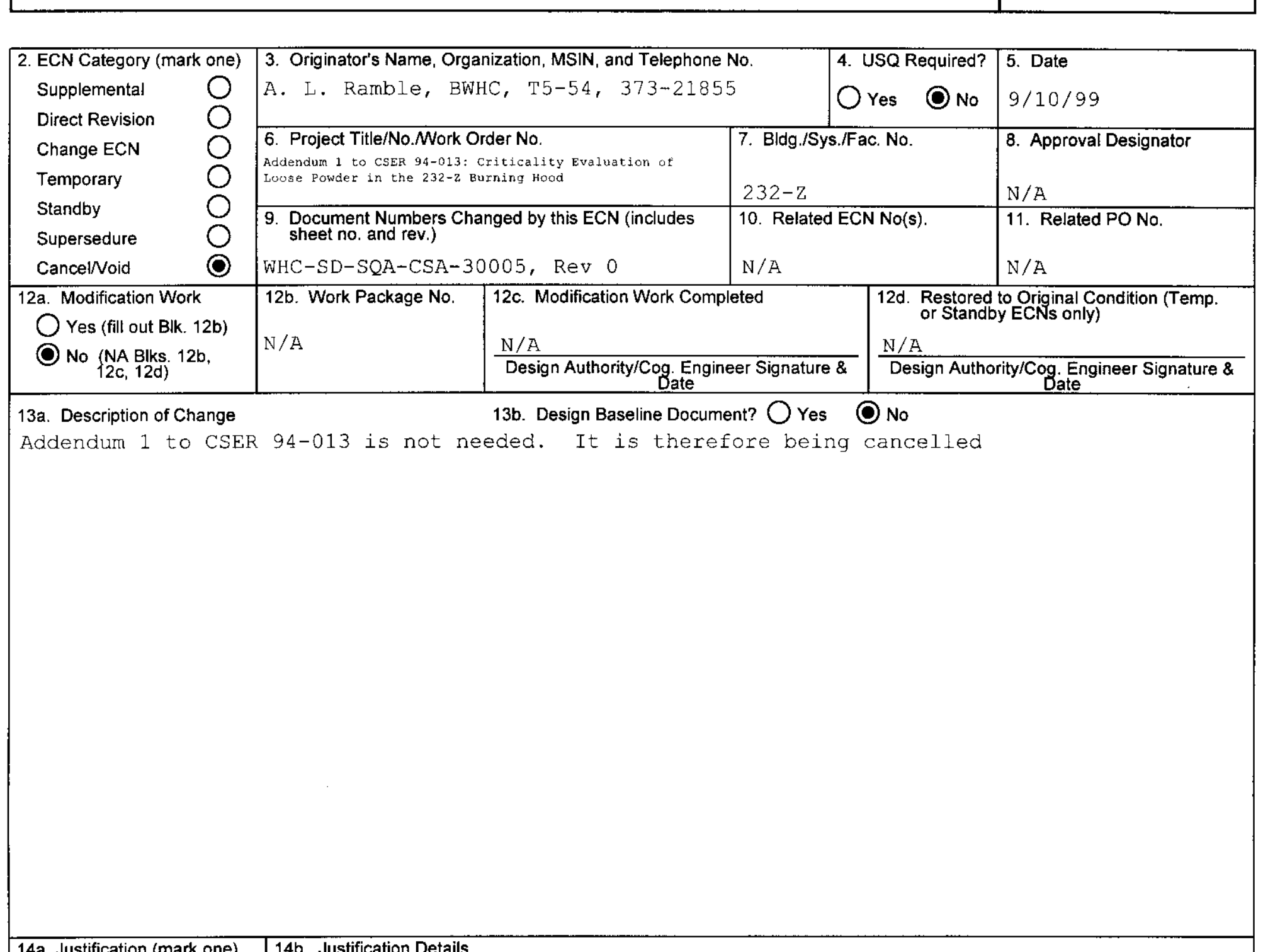

14a. Justification (mark one) $14 \mathrm{~b}$. Justification Details

Criteria Change O The CSER supported clean out of the 232-z Burning Hood and is no longer

Design Improvement $O$ needed. Cancellation of this CSER is directed by BWHC letter

Environmental $\bigcirc$ BWHC-15500-98-018.

Facility Deactivation

As-Found

0

Facilitate Const.

Const. Error/Omission

Design Error/Omission

15. Distribution (include name, MSIN, and no. of copies)

See attached Distribution List 


\section{ENGINEERING CHANGE NOTICE}

16. Design Verification Required
OYes
No

17. Cost Impact

ENGINEERING

Additional $\bigcirc \$$

Savings $\bigcirc \$$
Page 2 of 2

ECN (use no. from pg. 1)

654898

18. Schedule Impact (days)

Improvement

Delay

\section{CONSTRUCTION}

$\$$
Tank Calibration Manual

Health Physics Procedure

Spares Multiple Unit Listing

Test Procedures/Specification

Component Index

ASME Coded Item

Human Factor Consideration

Computer Software

Electric Circuit Schedule

ICRS Procedure

Process Control Manual/Plan

Process Flow Chart

Purchase Requisition

Tickler File

Fac. Proc. Samp. Schedule

Inspection Plan

Inventory Adjustment Request

Environmental Permit

20. Other Affected Documents: (NOTE: Documents listed below will not be revised by this ECN.) Signatures below indicate that the signing organization has been notified of other affected documents listed below.

\section{Signature}

Date

Design Authority M. E. Shaw T5-5077.5\%

Cog. Eng. A. L. Ramble T5-54 C

Cog. Mgr. P. E. Roege T5-57/ A/K KCh

QA

Safety

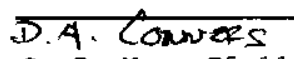

Environ.

Other

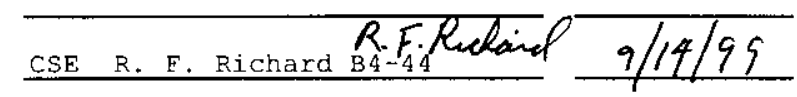

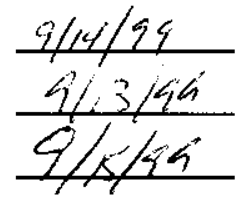

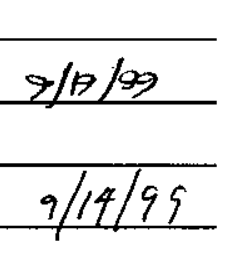

Signature

Date

Design Agent

PE

QA

Safety

Design

Environ.

Other

\section{DEPARTMENT OF ENERGY}

Signature or a Control Number that tracks the Approval Signature

ADPITIONAL 
$S$

HNF

INIXC-SD-SQA-CSA-30005, Rev. 1 Qterper

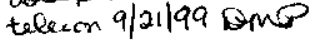

\title{
ADDENDUM 1 to CSER 94-013: CRITICALITY EVALUATION OF LOOSE POWDER IN THE 232-Z BURNING HOOD
}

\author{
A. L. Ramble \\ B\&W Hanford Company \\ Richland, WA 99352 \\ U.S. Department of Energy Contract DE-AC06-96RL13200
}

$\begin{array}{lll}\text { EDT/ECN: } & 654898 & \text { UC: } \\ \text { Org Code: } & 15500 & \text { Charge Code: } 101417 \mathrm{BB} 20 \\ \text { B\&R Code: } & \text { Ew7002010 } & \text { Total Pages: }{ }^{8} 2 \text { per al2lla9 } \\ & & \end{array}$

Key Words: 232-z Incinerator, PFP, Loose powder, Burning Hood

Abstract: This CSER is no longer needed so it is being cancelled

TRADEMARK DISCLAIMER. Reference herein to any specific commercial product, process, or service by trade name, trademark, manufacturer, or otherwise, does not necessarily constitute or imply its endorsement, recommendation, or favoring by the United States Government or any agency thereof or its contractors or subcontractors.

Printed in the United States of America. To obtain copies of this document, contact: Document Control Services, P.O. Box 950, Mailstop H6-08, Richland WA 99352, Phone (509) 372-2420; Fax (509) 376-4989.

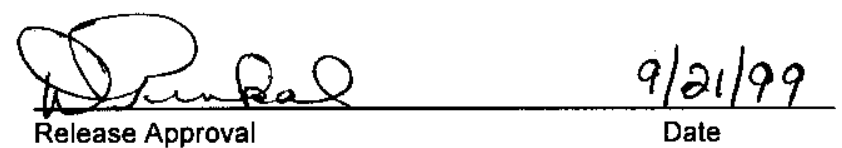

Release Approval

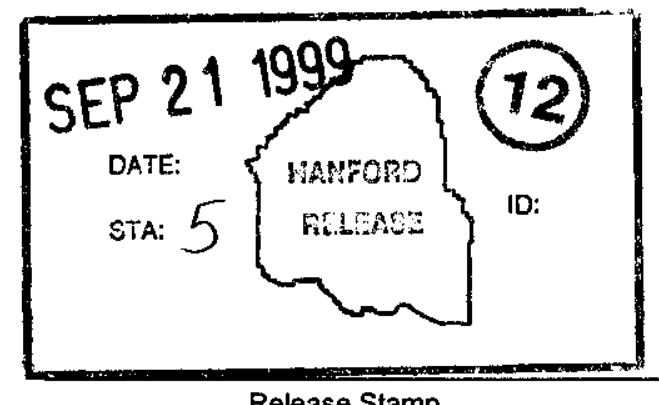

Release Stamp 


\section{RECORD OF REVISION}

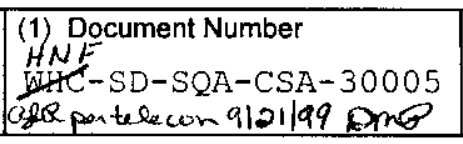

Page 1

(2) Title

Addendum 1 to CSER 94-013:Criticality Evaluation of Loose Powder in the 232-z Burning Hood

Change Control Record

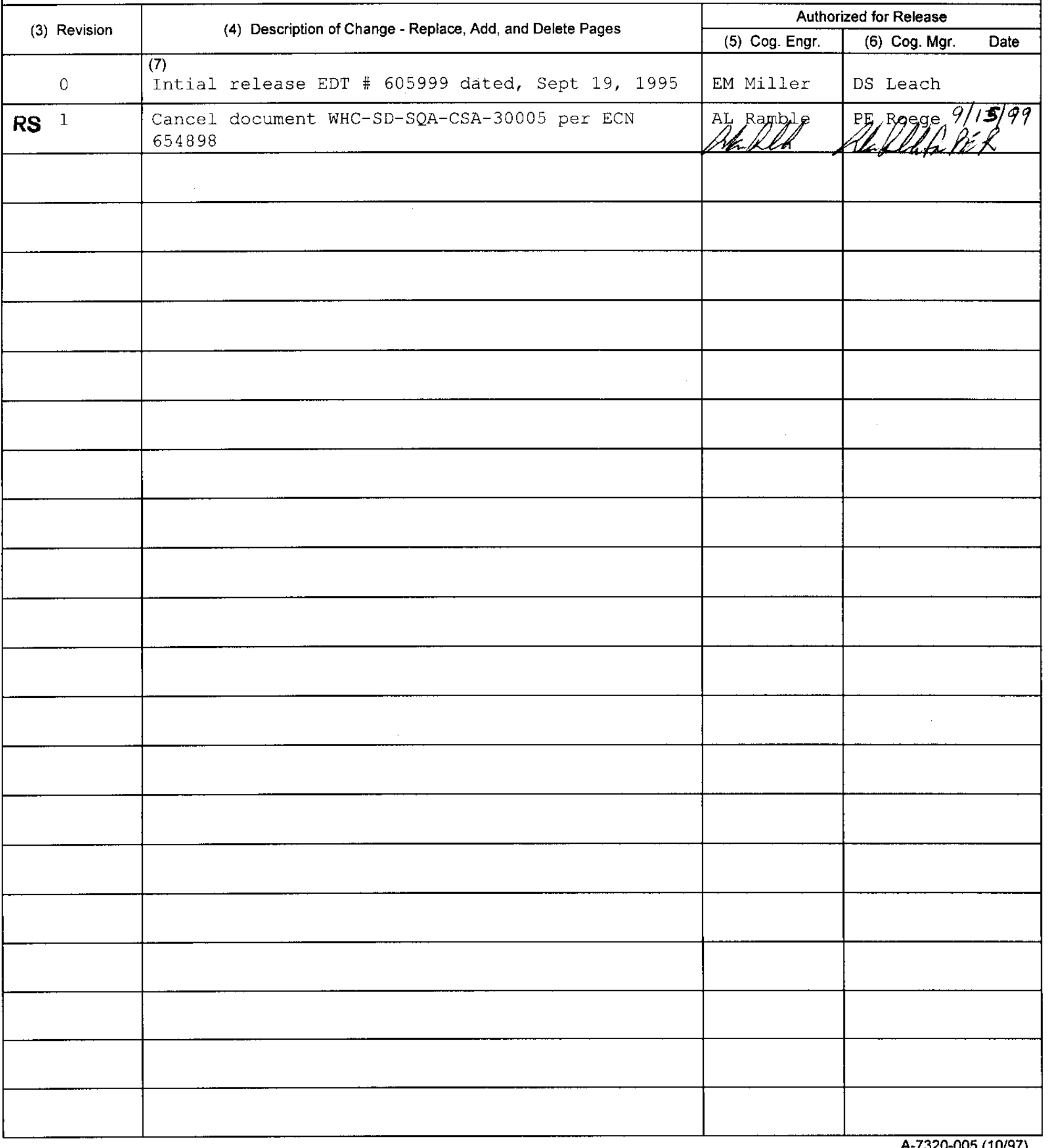




\section{DISTRIBUTION SHEET}

To

Distribution

\section{Project Title/Work Order}

Addendum 1 to CSER 94-013: Criticality Evaluation of Loose Powder in the 232-2 Burning Hood

\section{Name}

$\mathrm{B} \& W$ Hanford.

S.-E. Nuan D.A. Conners IV

A. I. Ramble

R. D. Redekopp

P. E. Roege

M. E. Shaw

DOE-RI

S. J. Altschuler

L. T. Nirider

Fluor Daniel Hanford

S. T. Almodovar

Fluor Daniel Northwest

K. D. Dobbin

E. M. Miller

R. F. Richard

H. Toffer

Central Files (Orig. +2)

Docket Files (2 copies)

\section{From \\ BWHC}

\begin{tabular}{|l|l|}
\multicolumn{1}{|l}{} & Page 1 of 1 \\
\cline { 2 - 3 } & Date $9 / 10 / 99$ \\
\cline { 2 - 3 } & EDT No. \\
\hline ECN No. 654898 \\
\hline Text
\end{tabular}

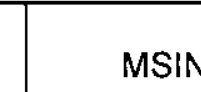

MSIN

\section{Tex}

With All

Attach.

$5-11$

T5-54

T5-15

T5-15

T5-54

\begin{tabular}{l|l} 
& \\
&
\end{tabular}

Attach.I

Appendix Only

$\mathrm{x}$

$\mathrm{x}$

$\mathrm{x}$

$\mathrm{x}$

$\mathrm{x}$

R $3-77$

A 5-55

$\mathrm{x}$

$x$.

A3-02

X

\begin{tabular}{l|l} 
A & \\
\hline & \\
\hline
\end{tabular}

\begin{tabular}{l|r}
\hline B4-44 & x \\
\hline B4-44 & x \\
\hline B4-44 & X \\
\hline B4 -44 & x \\
\hline
\end{tabular}

B 4-4 4

$x$

BI-07

A3-02
EDT/ECN Only 